\title{
MOMENT AND GEOMETRIC PROBABILITY INEQUALITIES ARISING FROM ARRANGEMENT INCREASING FUNCTIONS
}

\author{
By Philip J. Boland, ${ }^{1}$ Frank Proschan ${ }^{2}$ and Y. L. Tong ${ }^{3}$ \\ University College, Dublin, The Florida State University and \\ Georgia Institute of Technology
}

\begin{abstract}
A real-valued function $g$ of two vector arguments $\mathbf{x}$ and $\mathbf{y} \in R^{n}$ is said to be arrangement increasing if it increases in value as the arrangement of components in $\mathbf{x}$ becomes increasingly similar to the arrangement of components in y. Hollander, Proschan and Sethuraman (1977) show that the convolution of arrangement increasing functions is arrangement increasing. This result is used to generate some interesting probability inequalities of a geometric nature for exchangeable random vectors. Other geometric inequalities for families of arrangement increasing multivariate densities are also given, and some moment inequalities are obtained.
\end{abstract}

\section{Introduction.}

Definition 1.1. For a given vector $\mathbf{x}=\left(x_{1}, \ldots, x_{n}\right) \in R^{n}$, we let $\mathbf{x} \uparrow=$ $\left(x_{[n]}, \ldots, x_{[1]}\right)$ and $\mathbf{x} \downarrow=\left(x_{[1]}, \ldots, x_{[n]}\right)$ be, respectively, the vectors with the components of $\mathbf{x}$ arranged in increasing (decreasing) order. For any permutation $\pi$ of $\{1,2, \ldots, n\}$, we let $\mathbf{x}_{\pi}=\left(x_{\pi(1)}, \ldots, x_{\pi(n)}\right)$.

For vectors $\mathbf{x}, \mathbf{y}, \mathbf{u}, \mathbf{v}$ in $R^{n}$, we write $(\mathbf{x}, \mathbf{y}) \stackrel{a}{=}(\mathbf{u}, \mathbf{v})$ if there exists a permutation $\pi$ of $\{1, \ldots, n\}$ such that $\mathbf{x}_{\pi}=\mathbf{u}$ and $\mathbf{y}_{\pi}=\mathbf{v}$. We define $(\mathbf{x}, \mathbf{y}) \stackrel{a}{\leq}(\mathbf{u}, \mathbf{v})$ if there exists a finite number of vectors $\mathrm{z}^{1}, \ldots, \mathrm{z}^{K}$ such that

(i) $(\mathbf{x}, \mathbf{y}) \stackrel{\underline{a}}{=}\left(\mathbf{x} \uparrow, \mathbf{z}^{1}\right)$ and $\left(\mathbf{x} \uparrow, \mathbf{z}^{K}\right) \stackrel{a}{=}(\mathbf{u}, \mathbf{v})$, and

(ii) $\mathrm{z}^{i-1}$ can be obtained from $\mathrm{z}^{i}$ by an interchange of two components of $\mathrm{z}^{i}$, the first of which is less than the second.

Definition 1.2. A function $g$ of two vector arguments $\mathbf{x}$ and $\mathbf{y} \in R^{n}$ for which $g(\mathbf{x}, \mathbf{y}) \leq g(\mathbf{u}, \mathbf{v})$ when $(\mathbf{x}, \mathbf{y}) \stackrel{a}{\leq}(\mathbf{u}, \mathbf{v})$ is said to be arrangement increasing or AI by Marshall and Olkin (1979), and decreasing in transposition or DT by Hollander, Proschan and Sethuraman (1977). We shall use the terminology arrangement increasing (AI) in order to emphasize that such a function $g(\mathbf{x}, \mathbf{y})$ increases in value as the arrangement of components in $\mathbf{x}$ becomes increasingly similar to the arrangement of components in $\mathbf{y}$.

Received October 1985; revised April 1986.

${ }^{1}$ Research partially sponsored by AFOSR Grant F49620-85-C-0007 and AFOSR Grant 84-0113.

${ }^{2}$ Research sponsored by AFOSR Grant F49620-85-C-0007.

${ }^{3}$ Research sponsored by the National Science Foundation under Grant DMS-85-02346.

AMS 1980 subject classifications. Primary 60D05; secondary $62 \mathrm{H} 10$.

Key words and phrases. Arrangement increasing, decreasing in transposition, exchangeable random vector, family of arrangement increasing densities, inequalities, moments, Laplace transforms, permutation. 
Hollander, Proschan and Sethuraman (1977) have shown that the class of arrangement increasing functions is closed under some basic operations. In particular, the class of such functions is closed under mixtures, and the product of nonnegative AI functions is AI. If $\phi$ is an increasing function and $g(x, y)$ is $\mathrm{AI}$, then clearly $\phi(g(\mathbf{x}, \mathbf{y}))$ is AI. Perhaps the most powerful closure property they show, however, is that the convolution of $\mathrm{AI}$ functions is $\mathrm{AI}$.

Theorem 1.3 (Hollander, Proschan and Sethuraman). Let $u$ be a measure defined on the Borel subsets of $R^{n}$ such that for all Borel sets $A \subset R^{n}$ and all permutations $\pi, u(A)=u\left(A_{\pi}\right)$, where $A_{\pi}=\left\{\mathbf{y}: \mathbf{y}=\mathbf{x}_{\pi}=\left(x_{\pi(1)}, \ldots, x_{\pi(n)}\right)\right.$ for some $\mathrm{x} \in A\}$. If $\mathrm{g}_{i}$ is $A I$ on $R^{n} \times R^{n}$ for $i=1,2$, then the convolution $\mathrm{g}$ given by

$$
\mathbf{g}(\mathbf{a}, \mathbf{b})=\int g_{1}(\mathbf{a}, \mathbf{x}) g_{2}(\mathbf{x}, \mathbf{b}) u(d \mathbf{x})
$$

is $A I$, provided the integral exists.

We will illustrate some of the power of this result in Section 2 when we show that many geometric probabilities of random vectors $\mathbf{X}$ with exchangeable densities $f(\mathbf{x})$ are AI functions. In Section 3 we further illustrate this power with examples of geometric probability comparisons arising in families of AI multivariate densities. Some moment inequalities are also obtained.

2. Arrangement increasing functions from exchangeable random vectors. In this section we will assume $\mathbf{X}=\left(X_{1}, \ldots, X_{n}\right)$ is an exchangeable random vector [that is, the distribution of $\mathbf{X}_{\pi}=\left(X_{\pi(1)}, \ldots, X_{\pi(n)}\right)$ does not depend on the permutation $\pi]$ with density or mass function $f(\mathbf{x})$. Many interesting geometric probability functions which are arrangement increasing as well as some moment inequalities may be generated by the use of the following corollaries.

CoRollary 2.1. Let $\mathbf{X}$ be an exchangeable random vector with density or mass function $f(\mathbf{x})$. Let $h^{i}$ be an $A I$ function on $R^{n} \times R^{n}$ and $\phi_{i}=R \rightarrow R$ be nondecreasing for $i=1,2$. Then

$$
\psi(\mathbf{a}, \mathbf{b})=E_{\mathbf{X}}\left[\phi_{1}\left(h^{1}(\mathbf{a}, \mathbf{X})\right) \phi_{2}\left(h^{2}(\mathbf{X}, \mathbf{b})\right)\right] \text { is } A I \text { in }(\mathbf{a}, \mathbf{b}) \in R^{n} \times R^{n} \text {. }
$$

Proof. Let $g_{1}(\mathbf{a}, \mathbf{x})=\phi_{1}\left(h^{1}(\mathbf{a}, \mathbf{x})\right)$ and $g_{2}(\mathbf{x}, \mathbf{b})=\phi_{2}\left(h^{2}(\mathbf{x}, \mathbf{b})\right)$. Then $g_{1}$ and $g_{2}$ are $\mathrm{AI}$ (nondecreasing functions of $\mathrm{AI}$ functions are $\mathrm{AI}$ ). As $u(d \mathbf{x})=f(\mathbf{x}) d \mathbf{x}$ is a permutation symmetric measure, we deduce from Theorem 1.3 that

$$
\psi(\mathbf{a}, \mathbf{b})=\int g_{1}(\mathbf{a}, \mathbf{x}) g_{2}(\mathbf{x}, \mathbf{b}) f(\mathbf{x}) d \mathbf{x}
$$

is arrangement increasing in $\mathbf{a}$ and $\mathbf{b} \in R^{n}$.

COROLlaRY 2.2. Let $\mathbf{X}$ be an exchangeable random vector with density or mass function $f(\mathbf{x})$, and $h^{i}$ be an $A I$ function on $R^{n} \times R^{n}$ for $i=1,2$. Then for any two constants $c_{1}$ and $c_{2}$,

$$
P_{(\mathbf{a}, \mathbf{b})}(\mathbf{X})=\operatorname{Prob}\left(h^{1}(\mathbf{a}, \mathbf{X}) \geq c_{1}, h^{2}(\mathbf{X}, \mathbf{b}) \geq c_{2}\right)
$$

is an arrangement increasing function of $\mathbf{a}$ and $\mathrm{b} \in R^{n} \times R^{n}$. 
Proof. This follows from Corollary 2.1 by letting $\phi_{i}=I_{\left[c_{i},+\infty\right)}$ [the characteristic function of the $\left.\operatorname{set}\left[c_{i},+\infty\right)\right]$ for $i=1,2$.

EXAMPLE 2.3. The following are some elementary examples of AI functions which will be useful in illustrating Corollaries 2.1 and 2.2:

$$
\begin{aligned}
& \text { 1. } h_{1}(\mathbf{u}, \mathbf{v})=I_{\left\{u_{i} \leq v_{i}: i=1, \ldots, n\right\}} ; \\
& \text { 2. } h_{2}(\mathbf{u}, \mathbf{v})=\sum_{i=1}^{n} u_{i} v_{i} ; \\
& \text { 3. } h_{3}(\mathbf{u}, \mathbf{v})=-\sum_{i=1}^{n}\left(u_{i}-v_{i}\right)^{2} ; \\
& \text { 4. } h_{4}(\mathbf{u}, \mathbf{v})=-\sum_{i=1}^{n} \frac{u_{i}^{2}}{v_{i}^{2}} \times I_{\left\{u_{i}>0, v_{i}>0: i=1, \ldots, n\right\}} ; \\
& \text { 5. } h_{5}(\mathbf{u}, \mathbf{v})=\prod_{i=1}^{n}\left(u_{i}-v_{i}\right)^{+}, \quad \text { where }\left(u_{i}-v_{i}\right)^{+}=\left(u_{i}-v_{i}\right) I_{\left\{u_{i}>v_{i}\right\}} ; \\
& \text { 6. } h_{6}(\mathbf{u}, \mathbf{v})=-\max \left|u_{i}-v_{i}\right| ; \\
& \text { 7. } h_{7}(\mathbf{u}, \mathbf{v})=-\sum_{i=1}^{n}\left|u_{i}-v_{i}\right| .
\end{aligned}
$$

EXAMPLE 2.4. Judicious selection of $h^{1}$ and $h^{2}$ from, say Example 2.3, can yield some useful AI geometric probability functions. We illustrate this with some examples.

1. The rectangular probability

$$
\operatorname{Prob}\left(a_{i} \leq X_{i} \leq b_{i}: i=1, \ldots, n\right)=\operatorname{Prob}(\mathbf{X} \in[\mathbf{a}, \mathbf{b}])
$$

is an AI function of $\mathbf{a}$ and $\mathbf{b}$, as can be seen by using $h^{1}(\mathbf{a}, \mathbf{x})=h_{1}(\mathbf{a}, \mathbf{x})$ and $h^{2}(\mathbf{x}, \mathbf{b})=h_{1}(\mathbf{x}, \mathbf{b})$. [See also Boland (1985) for more on rectangular probabilities of this type.]

2. $\operatorname{Prob}\left(\sum_{i=1}^{n} a_{i} X_{i} \geq c_{1}, \sum_{i=1}^{n} b_{i} X_{i} \geq c_{2}\right)$ is $\mathrm{AI}$ in $\mathbf{a}$ and $\mathbf{b}$, as can be seen by letting $h^{1}(\mathbf{a}, \mathbf{x})=h_{2}(\mathbf{a}, \mathbf{x})$ and $h^{2}(\mathbf{x}, \mathbf{b})=h_{2}(\mathbf{x}, \mathbf{b})$. For example, if $\mathbf{X}=\left(X_{1}, X_{2}\right)$ is exchangeable, then $\operatorname{Prob}(\equiv)=\operatorname{Prob}\left(4 X_{1}+X_{2} \geq 4,2 X_{1}+4 X_{2} \geq 2\right) \leq$ $\operatorname{Prob}\left(X_{1}+4 X_{2} \geq 4,2 X_{1}+\overline{4} X_{2} \geq 2\right)=\operatorname{Prob}(|||| \mid)$. (See Figure 1.)

3. Of course, any combination of two of the types of functions in Example 2.3 gives us a probability function which is $\mathrm{AI}$ in $\mathbf{a}$ and $\mathbf{b}$, such as

$$
\operatorname{Prob}\left(\sum_{i=1}^{n} a_{i} X_{i} \geq c_{1} \text { and } \sum_{i=1}^{n}\left(X_{i}-b_{i}\right)^{2} \leq c_{2}\right)
$$

or

$$
\operatorname{Prob}\left(X_{i} \geq a_{i} \text { for all } i=1, \ldots, n \text { and } \sum_{i=1}^{n}\left|X_{i}-b_{i}\right| \leq c_{2}\right)
$$




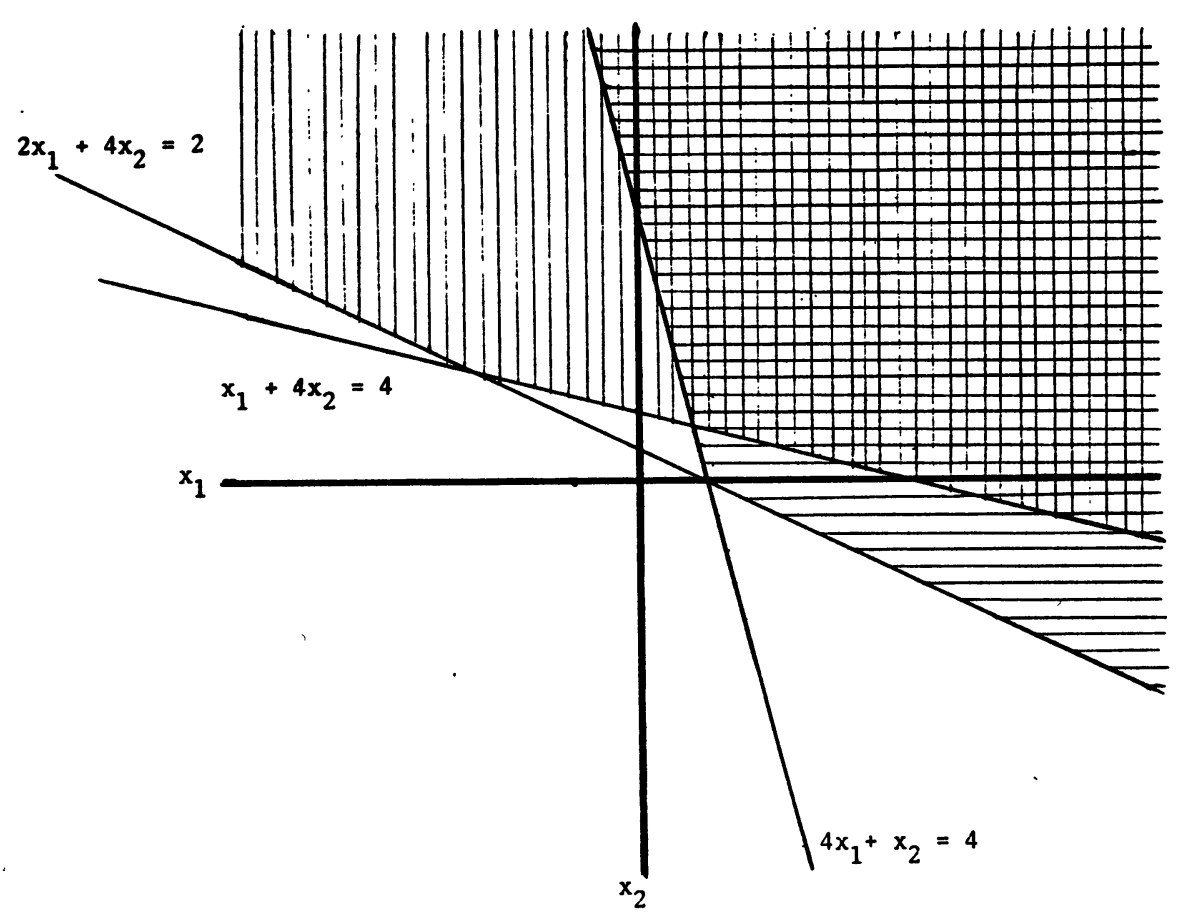

Fig. 1. Regions generated by AI functions.

Now let $\mathbf{X}$ be an exchangeable random vector with mass or density function $f(\mathbf{x}), h^{i}$ be an AI function for $i=1,2$, and assume $(\mathbf{a}, \mathbf{b}),\left(\mathbf{a}^{\prime}, \mathbf{b}^{\prime}\right) \in R^{n} \times R^{n}$ are given, where $(\mathbf{a}, \mathbf{b}) \stackrel{a}{\leq}\left(\mathbf{a}^{\prime}, \mathbf{b}^{\prime}\right)$.

We use the notation $\mathbf{Y}=\left(Y_{1}, Y_{2}\right) \equiv\left(h^{1}(\mathbf{a}, \mathbf{X}), h^{2}(\mathbf{X}, \mathbf{b})\right)$ and $\mathbf{Y}^{\prime}=\left(Y_{1}^{\prime}, Y_{2}^{\prime}\right) \equiv$ $\left(h^{1}\left(\mathbf{a}^{\prime}, \mathbf{X}\right), h^{2}\left(\mathbf{X}, \mathbf{b}^{\prime}\right)\right)$. Because $\mathbf{X}$ is exchangeable, $\mathbf{Y}$ and $\mathbf{Y}^{\prime}$ have the same marginals. In other words, the distributions of the marginals of $\mathbf{Y}$ are unaffected by a permutation of the components of $\mathbf{a}$ or $\mathbf{b}$, although their joint distribution may be altered.

REMARK 2.5. Corollary 2.2 says that $\left(Y_{1}^{\prime}, Y_{2}^{\prime}\right)$ is in a sense more positively quadrant dependent than $\left(Y_{1}, Y_{2}\right)$. See Lehmann (1966) and Barlow and Proschan (1981) for concepts of dependence.

We might say that the bivariate vector $\left(U_{1}^{\prime} ; U_{2}^{\prime}\right)$ is more positively dependent than $\left(U_{1}, U_{2}\right)$ if $U_{i}^{\prime}$ and $U_{i}$ have the same distribution for $i=1,2$, and

$$
\operatorname{cov}\left(\phi_{1}\left(U_{1}^{\prime}\right), \phi_{2}\left(U_{2}^{\prime}\right)\right) \geq \operatorname{cov}\left(\phi_{1}\left(U_{1}\right), \phi_{2}\left(U_{2}\right)\right),
$$

for every pair $\phi_{1}, \phi_{2}$ of nondecreasing functions. [See Rinott and Pollak (1980) for a slightly different concept of more positively dependent.] Then Corollary 2.1 implies that $\left(Y_{1}^{\prime}, Y_{2}^{\prime}\right)$ is more positively dependent than $\left(Y_{1}, Y_{2}\right)$ since for any 
nondecreasing $\phi_{1}$ and $\phi_{2}$,

$$
\begin{aligned}
& \operatorname{cov}\left(\phi_{1}\left(Y_{1}^{\prime}\right), \phi_{2}\left(Y_{2}^{\prime}\right)\right)-\operatorname{cov}\left(\phi_{1}\left(Y_{1}\right), \phi_{2}\left(Y_{2}\right)\right) \\
& =E\left(\phi_{1}\left(Y_{1}^{\prime}\right) \phi_{2}\left(Y_{2}^{\prime}\right)\right)-E\left(\phi_{1}\left(Y_{1}\right) \phi_{2}\left(Y_{2}\right)\right)
\end{aligned}
$$

REMARK 2.6. Corollary 2.1 yields moment inequalities when $\mathbf{X}$ is exchangeable taking values in $[0,+\infty)^{n}$. We use the notion of the previous remark except we now assume that $(\mathbf{a}, \mathbf{b}),\left(\mathbf{a}^{\prime}, \mathbf{b}^{\prime}\right) \in[0,+\infty)^{n} \times[0,+\infty)^{n}$ and $h^{1}$ and $h^{2}$ take nonnegative values.

Let $\phi_{i}(y)=y^{m_{i}}$ for $y \geq 0$ and $m_{i}$ a positive integer, $i=1,2$. Then Corollary 2.1 implies that when $(\mathbf{a}, \mathbf{b}) \stackrel{a}{\leq}\left(\mathbf{a}^{\prime}, \mathbf{b}^{\prime}\right)$,

$$
E\left(Y_{1}^{\prime m_{1}} Y_{2}^{\prime m_{2}}\right) \geq E\left(Y_{1}^{m_{1}} Y_{2}^{m_{2}}\right), \text { for all } m_{1}, m_{2} \geq 1 \text {. }
$$

3. Arrangement increasing probabilities for AI families of densities. Many families of multivariate densities $\left\{f_{\lambda}(\mathbf{x})\right\}$ have the property that the function $\phi(\lambda, \mathbf{x})=f_{\lambda}(\mathbf{x})$ is arrangement increasing in the parameter $\lambda$ and the outcome $\mathbf{x}$. The multinomial

$$
\begin{aligned}
\phi_{1}(\lambda, \mathbf{x})=N ! & \prod_{i=1}^{n} \frac{\lambda_{i}^{x_{i}}}{x_{i} !} \\
0 & <\lambda_{i}, x_{i}=0,1, \ldots, i=1, \ldots, n, \Sigma \lambda_{i}=1 \text { and } \Sigma x_{i}=N,
\end{aligned}
$$

and the multivariate normal distribution with common variance and common covariance

$$
\phi_{2}(\lambda, \mathbf{x})=(2 \pi)^{-n / 2}|\Sigma|^{-1 / 2} \exp \left(-\frac{1}{2}(\mathbf{x}-\lambda) \Sigma^{-1}(\mathbf{x}-\lambda)^{\prime}\right)
$$

[where $\Sigma$ is the positive definite covariance matrix with $\sigma^{2}$ on the diagonal and $\rho \sigma^{2}$ elsewhere and $\left.\rho>-1 /(n-1)\right]$ are but two such examples. For many other examples see Hollander, Proschan and Sethuraman (1977).

The following corollary of Theorem 1.3 enables one to construct many AI functions of a geometric type from AI families of densities.

CoRollary 3.1. Let $\left\{f_{\lambda}(\mathbf{x})\right\}$ be an AI family of probability densities (or mass functions), and assume $\mathbf{X}$ has density (or mass function) $f_{\lambda}(\mathbf{x})$. Suppose $h$ is an AI function and that $c$ is an arbitrary constant. Then

$$
P_{\lambda, \mathbf{a}}(\mathbf{X})=\operatorname{Prob}(h(\mathbf{a}, \mathbf{X}) \geq c)
$$

is an $A I$ function of $\lambda$ and $\mathbf{a}$.

Proof. This follows from Theorem 1.3 by letting $g_{1}(\mathbf{a}, \mathbf{x})=I_{\{h(\mathbf{a}, \mathbf{x}) \geq c\}}$, $g_{2}(\mathbf{x}, \lambda)=f_{\lambda}(\mathbf{x})$ and $u$ either Lebesgue measure or an appropriate counting measure on a discrete set.

We now illustrate Corollary 3.1 with diverse examples. 
EXAMPLE 3.2. We assume that $\mathbf{X}$ is a multivariate random vector with density given by $f_{\lambda}(\mathbf{x})$, and that the family $\left\{f_{\lambda}(\mathbf{x})\right\}$ is an AI family of densities.

1. $F_{\lambda}(\mathbf{a})=\operatorname{Prob}_{\lambda}\left(X_{i} \leq a_{i}: \quad i=1, \ldots, n\right)$ and $\bar{F}_{\lambda}(\mathbf{a})=\operatorname{Prob}_{\lambda}\left(X_{i}>a_{i}: \quad i=\right.$ $1, \ldots, n)$ are AI in $\lambda$ and a. [See Hollander, Proschan and Sethurarnan (1977).]

2. $\operatorname{Prob}_{\lambda}\left(\sum_{i=1}^{n} a_{i} X_{i} \geq c\right)$ is $\mathrm{AI}$ in $\lambda$ and a. As a special case, it follows that if $X_{i}>0$ for all $i$ and $\lambda$, then $\operatorname{Prob}_{\lambda}\left(\sum_{i=1}^{n} X_{i} / a_{i} \leq 1\right)$ is AI in $\lambda$ and $a$, where $\mathbf{a} \in(0,+\infty)^{n}$.

3. $\operatorname{Prob}_{\lambda}\left(\sum_{i=1}^{n}\left(X_{i}-a_{i}\right)^{2} \leq c\right)$ is $\mathrm{AI}$ in $\lambda$ and a. Hence for a given $\lambda$, the probability that $\mathbf{X}$ lies in a sphere of radius $\sqrt{c}$ with center $\mathbf{a}=\left(a_{1}, \ldots, a_{n}\right)$, increases as the order of the coordinates of a becomes more similar to the order of coordinates in $\lambda=\left(\lambda_{1}, \ldots, \lambda_{n}\right)$.

Similarly, it follows that

$$
\operatorname{Prob}_{\lambda}\left(\sum_{i=1}^{n}\left|X_{i}-a_{i}\right| \leq c\right) \text { and } \operatorname{Prob}_{\lambda}\left(\left|X_{i}-a_{i}\right| \leq c: i=1, \ldots, n\right)
$$

are both $\mathrm{AI}$ in $\lambda$ and $\mathbf{a}$.

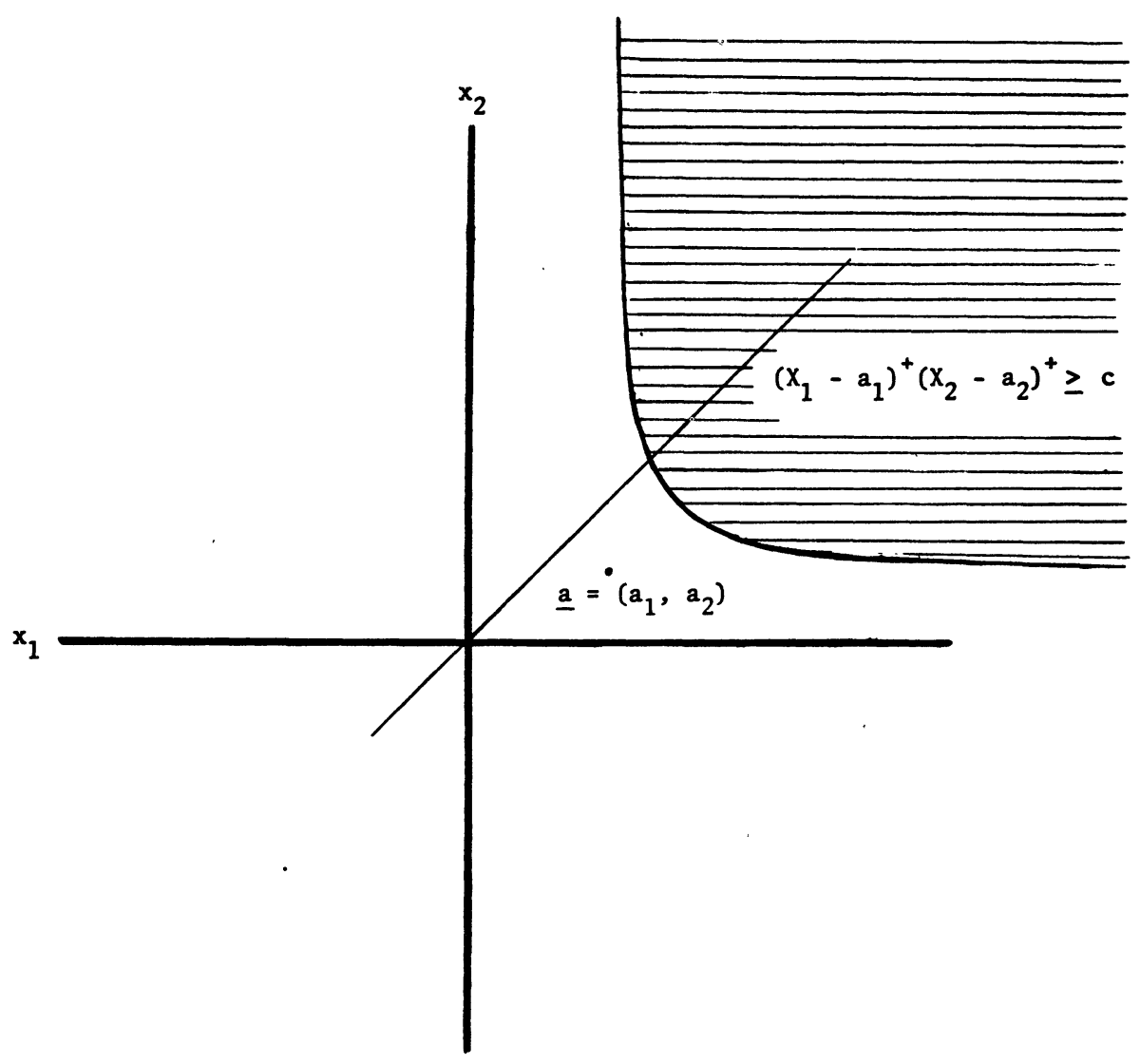

FiG. 2. 
4. If $\mathbf{X} \in[0,+\infty)^{n}$ with probability 1 for all $\lambda$, then $\operatorname{Prob}_{\lambda}\left(\sum_{i=1}^{n} X_{i}^{2} / a_{i}^{2} \leq c\right)$ is AI in $\lambda$ and $\mathbf{a}$ where $\mathbf{a} \in(0,+\infty)^{n}$.

5. $\operatorname{Prob}_{\lambda}\left(\prod_{i=1}^{n}\left(X_{i}-a_{i}\right)^{+} \geq c\right)$ is AI in $\lambda$ and a. The boundary of the above region for the two-dimensional case is a hyperbola, illustrated in Figure 2.

ExAMPLE 3.3. Suppose that $\left\{f_{\lambda}(\mathbf{x})\right\}$ is an AI family of densities, where each $f_{\lambda}(\mathbf{x})$ has support in $[0,+\infty)^{n}$. Now $\prod_{i=1}^{n} X_{i}^{m_{i}}$ is an AI function of $\mathbf{m}$ and $\mathbf{X}$, and hence a further application of Theorem 1.3 yields that $E_{\lambda}\left(\Pi_{i=1}^{n} X_{i}^{m_{i}}\right)=\mu_{\lambda}^{m_{1}}, \ldots, m_{n}$ is an AI function of $\lambda$ and $\mathbf{m}$. Similarly, $e^{\mathbf{x} \cdot \mathbf{t}}=e^{\sum_{i-1}^{n} x_{i} t_{i}}$ is also an AI function of $\mathbf{x}$ and $\mathbf{t}$. It is easy to see therefore that the multivariate Laplace transform (if it exists)

$$
M G F_{\mathbf{x}, \lambda}(\mathbf{t}) \equiv M_{\lambda}(\mathbf{t})=\int e^{\mathbf{x} \cdot \mathbf{t}} f_{\lambda}(\mathbf{x}) d \mathbf{x}
$$

is also an $\mathrm{AI}$ function in $\lambda$ and $\mathbf{t}$.

\section{REFERENCES}

Barlow, R. E. and Proschan, F. (1981). Statistical Theory of Reliability and Life TestingProbability Models. To Begin With.

Boland, P. J. (1985). Integrating Schur concave densities on rectangles. Unpublished manuscript.

Hollander, M., Proschan, F. and Sethuraman, J. (1977). Functions decreasing in transposition and their applications in ranking problems. Ann. Statist. 5 722-733.

Lehmann, E. L. (1966). Some concepts of dependence. Ann. Math. Statist. 37 1137-1153.

Marshall, A. and Olkin, I. (1979). Inequalities: Theory of Majorization and Its Applications. Academic, New York.

RINotT, Y. and PollaK, M. (1980). A stochastic ordering induced by a concept of positive dependence and monotonicity of asymptotic test sizes. Ann. Statist. 8 190-198.

Department OF STATISTICS

UNIVERSITY COLLEGE, DUBLIN

BELFIELD, DUBLIN 4

IRELAND

\author{
DEPaRTMENT OF STATISTICS \\ The Florida State University \\ Tallahassee, Florida 32306-3033
}

School of Mathematics

Georgia Institute of Technology

atlanta, Georgia 30332 